I found the left ventricle quite empty and firmly contracted, just as it has been found in cases of cholera. The right was occupied by a largish fibrinous clot extending up into the pulmonary artery. It did not appear of ante mortem origin. The lungs were congested. The absence of abdominal pain is not an argument against this view; as we know, from observation of epilepsy, that an unfelt irritation may give rise to very severe symptoms. Cases of this nature would be more amenable to treatment than others; inasmuch as the intestinal irritation might be removed by appropriate remedies, as calomel and opium, castor-oil, etc. It is to be feared, however, that, in many cases, the heart, kidneys, and other organs, are too severely stricken in their vital powers to carry on their necessary functions, even when the irritation of the intestinal nervous structures is removed.

In conclusion, I may say that I regard cholera as by no means a single and uniform morbid process, but one which consists of several varying elements. These may be enumerated as cardiac paralysis, constriction of arteries, alterations in the blood and capillaries, intestinal irritation. They may be variously developed in different instances, some may be more marked in one case, others in another, and remedial means should be varied accordingly.

\section{REMARKS ON SYPHILISATION.}

By George Gaskorn, Esq., Surgeon, Chevalier of the Order of Christ, Portugal; Surgeon to the Artists' Benevolent Fund; formerly House-Surgeon and House-Pupil, St. George's Hospital.

$$
\text { [Continued from page 41.] }
$$

Is the continuation of his second lecture, Mr. Lee says that the immunity obtained in animals through repeated inoculations was attributed by M. Auzias to saturation of the system with the syphilitic virus; and then ho goes on to say that "the early theory formed of the process was embraced by the word given to it; but that theory has perished, though the term has been maintained. Syphilisation is a misnomer. For, let us allow that we can make an indefinite number of ulcers on the person of a syphilitic by repeated inoculations of the pus of an indurated chancre, there is here no analogy to the process by which animal poisons are carried into the constitution-to vaccination, for example." Here we have it implied that Auzias Turenne has fled from the principles with which the public were first deluded and amused; he has experienced a defeat, we must suppose (for has he not shifted his ground ?); a loathsome mode of treatment is maintained, though the plea for its introduction is found to be groundless and untenable; "the theory embraced by the word given to it" is utterly destroyed; while, in obedience to necessity, the venue now is changed, and a source of professional notoriety is supported on some other ground of conclusion equally false and subvertible.

But why, oh ! Henry Lee, do you persist in laying your readers on a wrong scent by suggesting in athers the existence of untenable and varying opinions, for which there is only the very faintest foundation in truth? Why range yourself with the oppressors of a great discoverer, as if it were not plain to every eye that his tardily and meanly acknowledged merits are a reproach to the age in which we exist, even more than the false glitter of reputations which flourish by his side. Was it not truly said by the wicked lawyers of James's reign, Give us a line of writing from a man's hand; it shall be enough for us to hang him straightway? and you have got now this unfaithful record of a dream, you rake up this word "saturation", which, in conjunction with syphilis, expresses so revolting an idea. The intention and legitimate aim of the discoverer to regulate and destroy the action of the virus by means of the same virus, and thus to rescue the patient's health from destruction, is kept out of sight altogether; and we have this "saturation" presented to our notice, which is asserted to have been M. Auzias-Turenne's original conception in proposing a new treatment of syphilis ; and, "therefore", say you, "this process was naturally termed syphilisation-this theory has perished, while the term has been retained". But did this theory ever exist?"

See we not rather here revived the prejudices of the previous century, when to give a man the small-pox, or matter from out of the body of a cow, was called flying in the face of Providence, and marring God's likeness in his creatures, just as now we are accused of piling syphilis on syphilis ? What is your ground of warranty for this assertion? No greater than what we shall see to follow. In the earliest physiological communication made by $\mathbf{M}$. Auzias-Turenne to any learned society, November 1850 , in the brief summary of his crude ideas on syphilitic vaccination, or syphilisation, as he then commenced to call it, one phrase, or short section of an introductory character, runs simply thus. No. 3. "The noun-substantive syphilisation (the corresponding verb being syphilise) may be used to denote a sort of saturation of the living organs, or better a condition of immunity obtained by successive chancres"; and by and bye he says, in so many words, that the virus offers itself to us as the best means of contending with the difficulties of the disease.

Could anything be more modestly expressed by a youthful aspirant? But, just as any stick may serve to beat a dog, we see here how unfashionable talent may cry out in vain for protection against the imputation of absurdity; nay, even when one of the cleverest men and one of the best physiologists of the time is in question. If he be not buoyed up by a clique, he must go to the wall; and abroad, out of his own country, where his personal qualities and antecedents are unknown, he must be content to rank with a Hahnemann and a Paracelsus, or with smaller fry. You threaten the public with an accumulated syphilis; and ask, by implication at least, if Auzias-Turenne and the rest are to be allowed to fill up human creatures like beer-barrels, or swell them out like sponges, with syphilitic poison.

What can give a more sad idea of the times in which we live than to see favoured and diligent men like Ricord and Henry Lee, representative men of the day, putting themselves so entirely in the wrong with regard to the degree of merit to be found in their contemporaries? What a picture is thus afforded of the condition of science amongst us, when all sorts of forced interpretations, imperfect apprehensions, and explanations ill understood, weary out the thread of life and exhaust all philosophic patience! Such experience might seem to make a return to the middle ages desirable when ghostly charity attempered a material persecution, altogether not more terribly felt perhaps than this crushing prejudice from the regions of fashion, while the very organs of public liberty are made to swell the chorus of dispraise.

In what way, we ask, is syphilisation a misnomer? With a thorough-paced dualist, it is liable to such an interpretation, because he looks on the whole thing as a sham. Yet with no reason on his part. For these wise men of Iyons have lately decided 
that vaccinia is a wholly different disease from the small-pox. To use their hackneyed expression, it is "antagonistic" to it. Still none of them, that we have heard, affirm that the poison of vaccinia does not control small-pox. But Mr. Lee is not openly a dualist-as to difference of species, at least; he does not deny expressly to the matter of soft chancre the name of syphilitic matter; but if the cure is done with syphilitic matter, surely the patient is syphilised. The idea of making an indefinite number of ulcers is altogether assumed by Mr. Lee; it is on the definite limitation of the inoculations that the whole rationale is supported. The number of inoculations in a series and in the aggregate, though it may vary much, is necessarily limited; till, at last, when the final limit is reached, the most erosive virus no more affects the frame than a drop of water laid upon the skin. What does the writer know about the process by which animal poisons are carried into the human system? Does he know more than the rest of the medical world? Has he any particular theory to support? Is there one path, one process for them all? We know little enough about these things as yet.

As regards vaccinia, then, the poison does enter the constitution; we should scarce have expected such a confession from Mr. Lee; we should have thought he would have declared the operation of the vaccine vesicle to be "localised", "its history terminating with its cicatrisation." What proof to the contrary, if we omit its protective power? In the soft chancre, beside other proofs, there is a curative power as regards syphilitic disease. This is one of the reasons why we believe it affects the constitution, and is not a purely local or "localised" sore, the sphere of whose operation, according to the Lyons school, is confined, "like a spine in the tissues," to the neighbourhood of its site; and we also believe its secretion to be of a syphilitic nature and origin, so that, whether matter from soft chancre or hard chancre be used, syphilisation is not a misnomer.

But, forasmuch as no imputation can be more serious than that of thrusting back science and impeding the freedom of its career, we may say that we agree with that admirable writer, Bassereau, as to the existence of a simple ulcer of the genitals before the advent or cataclasm of syphilis in Europe; but it is not this Venerola which we have to deal with in the present day, but a soft chancre, which is a product of syphilis, and which is syphilis in a modified or fluctuating form, just as it falls under observation when we inoculate, and such as is signified, apparently, in the pages of Guicciardini, where this writer says that in the course of his not very lengthy experience, which dated from the very beginning of the French disease in Italy; it had become mitigated, " having divided spontaneously into more than one sort differing from what it was at first"; and too much weight cannot be given to his words. Although the history of the soft sore is the most obscure problem in the study of syphilis, this is what we are inclined to believe concerning it : we think ourselves justified in considering it to be part and parcel of the disease; and we believe that Bassereau's view of the subject is a partial one, for he has not taken all the facts into consideration.

As to the thrust of the indefinite number of hard chancres, we may say that the sophism is of Paris importation. It is repeated, as regards the soft sore in all new French treatises, as well as in the books which are copied from them. It is a truth which cannot be denied, that of all mankind the Parisians are the most gullible; for, if the Londoner enjoy a standing testimonial of his credulity, having acquired the name of cockney, yet the Parisian, as far more sangaine, unballasted, fed on silly vanities, goes very far beyond him in this respect, so as indeed to have merited a more significant epithet which it will not be necessary to mention.

Now, Lindman, the young German physician, who was one of the first to try multiple inoculations on himself for syphilis, when his fellows at the Académie de Chirurgie, fresh from their masters' teaching, came round him pitying his condition, and beseeching him to desist, told them people would never believe that syphilisation was so bad as in truth it was, until some worthy and excellent man had died of it, and therefore he had determined to go on. His subsequently improving health and flourishing appearance being the subject of general remark, defeated their anticipations of his decease. He still went on inoculating; and he appears to have told some one who questioned him or bothered him (for he would have nought to do with the Academy after their first behaviour to him), that he had inoculated himself with 2200 chancres, and after that he had ceased to count. He seems to have taken a pretty exact measure of their credulity, and to have reproved with a just sarcasm their pretentious scepticism. It is necessary to say that the fact of so many inoculations is perfectly absurd. The greatest number got by Boeck out of a large collection of cases treated by him was not a third of the number mentioned. How few of them are ulcers, it is not necessary to describe. The exaggeration of the French shows how far their wishes extend beyond their proofs in propping up their artificial systems.

This story is on a par with that still current in the Quartier Latin, of a student, J-, killed by syphilisation, a piece of tragedy retailed to a friend of ours, some time since, by a medical bookseller of the Quartier, with the true accent de la vérite. The tale occurs in the celebrated Lettres sur la Syphilis. The more correct version is as follows. Abont fifteen years gone by, a young student, in very distressed and unfortunate circumstances, adding toil to dissipation, died of contagious erysipelas, then raging in the house and quarter, at a time when the few inoculations that had been made on him were (as we have it on the evidence of M. Auzias-Turenne) wholly cicatrised.

M. Ricord, in a book characterised rather by " humorous anecdotes" and "situations" than by anything like fair argument, applies to this event his favourite word "deplorable" less in pity than in cen. sure; and the case is still quoted as a warning to all who dare, even in thought, oppose themselves to those who hold the reins of power in the schools of science; and, since the action of animal poisons is in question, this would seem the best opportunity of referring to that argument which Mr. Henry Lee has put forward in his last lecture, as to the immunity which is attained after bites of fleas and mosquitoes; either constantly reiterated or multiplied at short intervals of time. Whether an effort of candour prompted our objector to a confession which ho makes so entirely for us, or whether, as we rather believe, he brings it in to support a theory of deriva. tion which we entirely reject, it would still appear to range itself under the head of those stories to which we have referred above. Such a tale seems to want confirmation from popular experience. We have inquired of great travellers and others who have been much among those things, and they have answered us with smiles, or with laughter not unaccompanied with tears. But if he have persuaded himself of this matter, we may surely be permitted to give attention to what we have heard concerning 
immunity obtained by repeated inoculation of the poison of serpents, a far more important fact, but which, as far as we are concerned, needs further elucidation, though the sources from which we heard of it are more than respectable.

M. Ricord may proffer and publish the belief that syphilis is a degraded glanders, M. Diday expect to find in vaccine matter the materials for a cure; English syphilographers may depend on chlorate of potash, and give sulphite of soda a fair and lengthy trial ; but when a method of treatment offers itself, which is in accordance with pathology, and an order of ideas are produced which rest on instruction, observation, and experiment, it is decried as baseless and absurd, and its adrocates, too, are treated with slight respect, if not with scorn and inattention.

So far from there being no analogy between syphilisation and the operation of animal poisons, it is by analogy that we are guided throughout. Without this guide to the practitioner, we think it scarcely right and hardly possible to expect much success. The attention of modern physicians has been far less directed to the class of virulent diseases than formerly. Of late, pneumonia, carditis, diabetes, pyæmia, rheumatism, and other complaints, have excluded fevers and virulent diseases from much notice. Only fitfully and exceptionally have they engaged attention. It was far otherwise in the middle of the eighteenth century. When inoculation for small-pox arrived in England in the year 1721, although its inventor was lost to view, it came soon to be considered one of the noblest efforts of the human mind. We know the difficulties opposed to it; and that thirty or forty years had passed away before its merit was well recognised. From that time onward, every one's head was working in one direction. Inoculation was the order of the day ; for the cure of syphilis, it was first tried in the year 1778. This idea with regard to syphilis obtruded itself on many minds. The circumstance of the disease running itself out, and of its being an eruptive disorder, made the analogy too obvious not to occur to many. The chief objection to the treatment lay in the chronic character of the disease, so dif. ferent from small-pox and other exanthems, which pervade the tissues rapidly.

Before the discovery made by Auzias-Turenne, in the middle of our century, the medical mind of Paris had been deeply stirred by discussions on the occurrence of glanders in man. It required the stubborn perseverance and earmest soul of Rayer to prevent this pathological fact being consigned to the grave of the Capulets. The evidence of cases went for nothing against an opposition of the most bitter kind. All the questions which relate to the contagion and course of virulent diseases came under review and consideration at that hour; and it was under the impulsion of ideas which had become familiar through discussion, that Auzias-Turenne availed himself of opportunities he then possessed to attempt the inoculation of animals with syphilis. Being fresh from anatomical study, a delicate and dexterous manipulator, and withal earnest in the work, he succeeded, where Hunter, Ricord, Cullerier, with a less degree of earnestness, had failed. When M. Auzias-Turenne had discovered a natural limit to the number and extent of the inoculations, the path was traced out to that point of success which was uppermost in his mind, but which had equally suggested itself to others. Immunity was found at length to be attainable. Mankind were now suffciently well informed to put the plan in execution; and, as it appears to us, it has been followed by a certain measure of success.

That Dr. Boeck inoculated, or caused to be incontated, the hard chancre on the bearer during his stay in England, admits not of a doubt; that the same has been done since his departure cannot be denied. Mr. Lee believes he can inoculate and get a soft sore with an unwashed lancet wiped with dry lint, such as is commonly done by Dr. Boeck in his clinical practice. This, however on the first blush it may appear plausible, has not been put to the test by the objector.

In writing of the inoculable pus obtained from stimulated chancres, Mr. Lee expresses himself thus: "Whether," he says, "such matter be a product of true syphilis, or whether it depends on some dynamical change in the action of the part, it is essentially different from that which pertains to an infecting sore." This secretion, whether theoretically we sup. pose it to have its origin in syphilis or not, is practically not that which produces syphilis. In the change of action which has taken place during its production, it has lost its sting so far as its power of producing an indurated chancre is concerned, and it has also lost, so far as present evidence goes, its power of affecting the patient's constitution." Our foregoing argument will give us the key to such errors as these. He holds to the false belief, that an inoculation on a syphilitic soil carries no principle of infection; and he is then driven into a corner to find what can have happened in the sore. "It is changed," "it is essentially different," it is " practically different," "it has lost its sting-the sting which it has in the parent sore." An unicist would look on this with calmness; but to a dualist of the advanced school it is very perplexing.

Of the disappearance of the virus $\mathrm{Mr}$. Lee is fully convinced. The theories of Langlebert are so flattering in their application; the girls from the clinique of Bidenkap shut out all else from view. But what if there be no change after all? What if such sores do infect ?* This, truly, is what Mr. Lee will be the last man to allow, because it gives a shock to his system; for he has spent many years in constructing an "edifice", and never was there a more ingenious artificer than our friend. His edifice, however fairly built, will have to share the fate of many modern constructions. The keystone of this fair building is " adhesive inflammation", as distinguished from "suppurative ac. tion"-a secretion of sero-epithelial débris, in antithesis to pus. But is not the distinction between lymph-globule and pyoid globule too subtle whereon to establish a difference in kind, or, let us say, in species? In fact, the difference between them is roundly denied by the French, with whom the chancrous secretion, in all its forms, is pus. Fibroplastic globules and fibres have been somewhere called, we believe, heteromorphous; and the pyoid globule, homoiomorphous; but the line was never cut so sharp between them as we find it in this artificial theory before us. Nor, indeed, is a pusglobule, according to our ideas, exactly a pathological entity. To Henry Lee, who identifies the infecting sore with adhesive inflammation, the passage, whether by artificial or other means, to a suppurating sore, is all-important; it is an "artificially induced" action, manifested in the pus, and conveyed by the pus. The sore has lost its sting; it has "lost its infecting principle; and it has gained a contagious principle, as a result of this action. What does all this mean? It conveys the idea that there has been produced a soft sore, a contagious non-infecting sore and mark, not in situ where the "action" has been "induced" by irrita-

* See case of Melchior Robert's pupil, quoted in previous paper. Ricord has stated that, if chancrous pus be inoculated on a syphilitic person, the sore produced is never an indurated ulcer; but, notwithstanding, if the pus from it be transplanted to a non.syphiilitic, a hard chancre is the result, and also consecutive syphilis.
Seo also Bumstead On Venereal Diseases, 1864, Introduetion, p. 46. 
tion of some sort; bat manifested in the spot of inoculation, where the infecting power is lost, "as far as present evidence goes," and the contagious power is retained; so that from this sore you can inoculate right on in a series, although it be no longer pox.

Wrong in fact, wrong in theory. The case of the pupil of Robert, who, as we have seen, was infected through one of these inoculations, shows that it has not necessarily "lost its sting"-i.e., its infecting power. But let us follow Lee in his argument, in which he so nicely distinguishes contagious from infecting power. It needs explanation truly, and one requires to be behind the scenes. Let us consult his French prototypes. According to their purely artificial distinction, contagion is local, infection general in its effects. A small quantity of blennorrhagic pus deposited on a mucous surface, followed, as often is the case, by the disease, marks blennorrhagia as having a contagious principle. The same term, then, applies to the simple or soft venereal sore, which is with the French a local, contagious, non-infecting disorder. We now understand what he means by its losing its infecting, and gaining a contagious power. He means, totidem verbis, it has ceased to be an infecting, and has become a soft or simple chancre. There has taken place a change of species ; that is what he insinuates. This is the theory of the dualists and of Langlebert, undisguised. But how does this change occur, and when? At what point? During its flight to the inoculated part; at the moment of departure or of arrival. One disease goes out, and the other comes in, like two sentinels relieving one another, or night-nurses taking their turn about. But this contagious sore, whence comes it? Not out of the savine powder or the dry lint of the manufactory? Does it come $e$ nubibus, for ex nihilo nihil fit? It is the result, the author says, of "action"; it is "action" which is at the bottom of the change, and which produces a sore which is not a common ulcer, but which can be inoculated right on in a series. The passage from adhesive inflammation to suppurative inflammation, is it so extraordinary that it makes quite a change of disease? The action does not only break down the syphilitic principle completely, but it manufactures out of it an entirely now article, a most definite non-syphilitic malady, which, if we may believe the dualists, had a preexistence to syphilis among European populations. But perhaps Mr. Lee may say it is not a disease at all which is begotten; it is an "action" which is " induced". Does not common sense revolt at that? Suppose now you were to say to a man, "Sir, excuse me, you have not a disease hanging about you ; you have an action." Might he not answer, "Sir, I never had'an action in all my life? I have really something that ails me." Would it not be a scene de Molière? No, that argument will not do. Would it hold good in pneumonia, phlegmon, or any disease? Does leprosy begin only in a tubercle, and not also in an ulcer or a scale? Are we then so precise? We have heard something of polymorphism lately, and the term has been applied with a somewhat lavish hand to affections which are tolerably regular in their course; but syphilis, has it not always been esteemed polymorphic par excellencethe polymorphic disease? $\mathrm{Be}$, therefore, less exacting as to its elementary forms and primary deviations, we beseech you.

It remains for us to add, that a strong corroborative proof is given of the infecting character of the inoculations in the occurrence of the multiple glandular swellings which occur "like a chaplet" on the edge of the pectoral muscle, as described by Boeck and Bidenkap; and the same may be said of those skin-manifestations so convincing to the mind of the operator that the inoculated virus really has what $M$. Rollet and others have been pleased to deny to the simple sore-an "echo" in the economy.

$$
\text { [To be continued.] }
$$

We are requested to remind members of the Association that the Annual Subscriptions becarre due on the 1st of January. They can be paid either to the Secretaries of the Branches; or to the General Secretary, T. Watkin Williams, Esq., 13, Newhall Street, Birmingham.

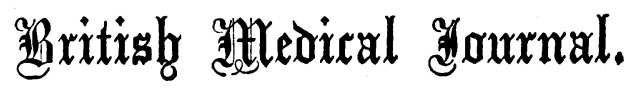

SATURDAY, SEPTEMBER 1st, 1866.

\section{A P HASIA.}

A GREAT deal of very good work has recently been done, chiefly in France, in the attempt to fix the seat of the faculty of language or of articulate language. We believe, for our part, that it is not well to speak of language as being a distinct faculty-at all events, in the sense of its being so distinct from the rest of mind and its outward doings as to have a separate seat in a small part of the brain. There has been much discussion on the subject; but, to our thinking, the work which will last will be that which M. Broca and others have done in collecting facts to shew where the Nervous System is most often damaged in cases where power of speech is lost or disordered. It may seem at first glance to be a tiresome refining of words to speak as if it were settled-although we do not say that we admit even this-that damage within a limited region of the nervous system disorders or destroys speech, whilst we will not admit that language has a seat. Within the limits of this short article, we have no intention whatever to discuss the subject of language; nor, in speaking briefly, must we be understood to wish to dogmatise on points which are very much disputed. Our object is rather to contribute what we can to greater width and precision of investigation, and especially to point out that the distinction we have just indicated is an intelligible one.

In many published cases of loss of speech, the damage to the nervous system has been of convolutions in the region of the corpus striatum; and, according to M. Broca, it has generally involved part of the third left frontal convolution. It nearly always involves the corpus striatum too, and hence loss or defect of speech is almost invariably attended by hemiplegia. Speaking of those cases in which the damage is the result of embolism, a writer in a contemporary (Lancet, Nov. 26th, 1864) says :

"In some, the supply of blood is cut off from so large a quantity of brain round about the highest 